\title{
Illustrating Transparent Surfaces with Curvature-Directed Strokes
}

\author{
Victoria Interrante \\ ICASE, NASA Langley Research Center
}

\author{
Henry Fuchs and Stephen Pizer \\ University of North Carolina- Chapel Hill
}

\section{ABSTRACT}

Transparency can be a useful device for simultaneously depicting multiple superimposed layers of information in a single image. However, in computer-generated pictures as in photographs and in directly viewed actual objects - it can often be difficult to adequately perceive the threedimensional shape of a layered transparent surface or its relative depth distance from underlying structures.

Inspired by artists' use of line to show shape, we have explored methods for automatically defining a distributed set of opaque surface markings that intend to portray the three-dimensional shape and relative depth of a smoothly curving layered transparent surface in an intuitively meaningful (and minimally occluding) way.

This paper describes the perceptual motivation, artistic inspiration and practical implementation of an algorithm for "texturing" a transparent surface with uniformly distributed opaque short strokes, locally oriented in the direction of greatest normal curvature, and of length proportional to the magnitude of the surface curvature in the stroke direction. The driving application for this work is the visualization of layered surfaces in radiation therapy treatment planning data, and the technique is illustrated on transparent isointensity surfaces of radiation dose.

\section{MOTIVATION}

There are many applications in scientific visualization that require or can benefit from the simultaneous display of multiple superimposed layers of information, and transparency can be a useful device for this purpose. The challenge is to render the transparent surfaces in such a way that they can be both clearly seen and easily seen through at the same time.

Photorealism, while a worthy goal, does not provide a sufficient or necessarily appropriate solution to this problem. The disciplines of medical and scientific illustration flourish today precisely because it is so often the case that a photograph cannot adequately convey the necessary information about a subject or scene [17]. With artistic enhancement, one has the potential to minimize extraneous detail and emphasize important features in order to portray information more clearly [11].

In order to determine how best to enhance the comprehensibility of a transparent surface, we begin by reviewing the factors that make the shape and relative depth of transparent surfaces so difficult to see.

\section{PERCEIVING THE SHAPE AND DEPTH OF A TRANSPARENT SURFACE}

Many of the most perceptually significant cues to shape and depth are not available on transparent surfaces. Shape-from-(diffuse) shading information is minimal at best, and occlusion, ordinarily one of the most powerful indicators of depth order relations, is present only where the high intensity of the reflected light at the specular highlights precludes the discrimination of underlying objects. The effects of refraction, which are strongly apparent in the regions of a surface most nearly aligned with the viewing direction, emphasize the silhouette and contour regions of a transparent form but at the expense of distorting the appearance of underlying structures. As our objective is to represent superimposed layers of information in such a way that each surface can be easily understood, the effects of refraction may do more harm than good. In this work we have chosen not to model the effects of refraction and to rely on luminance differences to convey the figure/ground discontinuities.

Specular highlights, because their locations on a surface are viewpoint-dependent, are less-than-ideal indicators of surface depth - under both monocular and binocular viewing conditions.

In a stereo view, specular highlights will appear to float slightly above or below a curved surface rather than to lie on it. Figure 1, after [2], illustrates this concept.
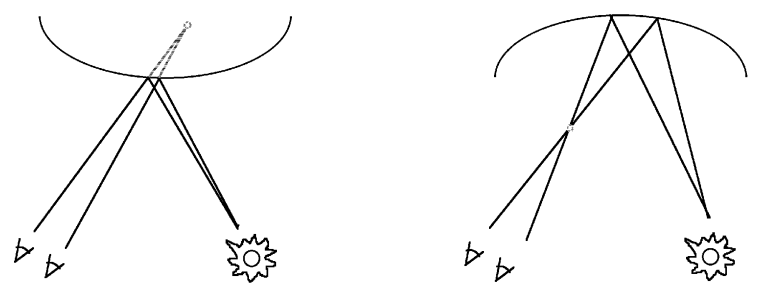

Figure 1: This diagram, modeled after a similar image by Blake and Bülthoff [2], describes why specular highlights will appear to float behind convex surfaces and in front of concave ones.

Although psychophysical evidence indicates that, with minimal practice, people can learn to use the direction of the depth disparity between the surface and the specular highligh to disambiguate aspects of the surface's shape [2], 
there is little reason to believe that specular highlights can meaningfully indicate surface depth in a stereo view - the extent of the apparent displacement varies as a function of both the viewing distance and the magnitude of the surface curvature.

As an object is moved about or viewed from a different position, the apparent locations of the specular highlights on (or near) the surface will change. Although the patterns of motion of specular highlights across a curved surface can provide a limited amount of shape information [14][33], the relative extent of the motion of these highlights can only be clearly perceived when reference features such as those provided by optical texture are available on the surface [2], and little depth information is given either by the velocity gradients of the specular highlights or by their relative motion, in the case of a superimposed transparent surface, with respect to the locations of specific points on an underlying opaque object.

There are a number of reasons to believe that we might be able to facilitate the perception of both the shape and depth of a layered transparent surface, without unduly impairing the visibility of underlying objects, by "artistically enhancing" the transparent surface with a small, stable set of opaque markings. Psychophysical experiments have shown that people perceive more depth in images in which complementary information is provided by multiple cues [4]; rather than being redundant, these repeated indications of similar information appear to reinforce each other, strengthening the overall depth perception [32]. By fixing a sparse, opaque "texture" on a transparent surface we can specify its three-dimensional location more explicitly, provide stable occlusion cues, introduce edges that may facilitate the veridical perception of depth from binocular disparity, and define fixed surface points whose velocity gradients under object or observer motion can better convey kinetic depth information.

\section{BACKGROUND}

In a previous paper [13], we described the perceptual motivation for using valley and ridge lines as an artistic device for communicating the essential shape features of familiar objects. Not all surfaces, however, can be easily characterized by prominent shape-based features and, additionally, there are situations in which we desire a more continuous representation of a transparent surface that encompasses both areas in which the surface shape is changing and areas across which it remains relatively uniform.

It has long been recognized that shape may be conveyed more effectively by the addition of texture elements to a surface [28], and the idea of using opacitymasking texture to enhance the visibility of a transparent surface is also not new. The three basic approaches that have been previously proposed include: texturing the projection of a transparent surface in the image plane using a screen-space opacity mask [6]; using a solid texture [20] to define a uniform "grid" that can be applied as a volume opacity mask [15]; and using conventional texture-mapping techniques [3] to apply a procedurally-defined two- dimensional texture as an opacity mask across a surface in 3D [23]. Similarly, the representation of a continuous surface by a set of discrete elements such as points, lines or other graphical primitives has a long history in many applications that use computer graphics for interactive data display [5][21][24]. Originally designed more for the purpose of rendering efficiency than to improve the comprehensibility of surface shape, it can be argued that the use of these primitives actually serves both purposes quite well.

The particular contribution of the work presented in this paper is to suggest that the shape and depth of a transparent surface might be communicated particularly effectively via a sparse opaque texture that is explicitly designed to convey intrinsic surface shape properties in a perceptually intuitive, orientation-invariant and geometrically meaningful way.

\section{ARTISTS' USE OF LINE TO SHOW SHAPE}

Although circular texture elements may individually describe local surface orientation in a particularly effective manner [29][1], they are rarely employed by medical and scientific illustrators as a device for portraying surface shape. Rather, artists and illustrators appear to rely on the use of shading and stroke direction to represent the threedimensional form of a surface in a two-dimensional image. For a number of reasons we would like the definition of the surface texture to be viewpoint-independent, and this precludes explicitly using texture properties to represent surface shading (although shape-from-shading information will continue be available in the illuminance distribution across the opaque texture elements).

The importance of stroke direction in line drawings has been repeatedly emphasized by artists [30][22][9]. Several different basic techniques are commonly used to define local line orientation, and it is popularly recognized that our perception of a surface's form will be affected by the choice of line direction used to represent it. When strokes are applied in a uniform direction across an entire image, objects tend to appear "flattened"; vertically-oriented strokes emphasize height and horizontally-oriented strokes emphasize width [22]. Stroke direction can also be defined by the "fall of light upon the object" [30], represented as concentric arcs or radial lines emanating from a point light source, but this approach too can have a flattening effect, particularly where the orientation of the strokes closely parallels the boundary of the form [30]. A third approach, and the method that appears to most closely describe a common use of line by medical illustrators, is to align the stroke direction with the direction of strongest curvature of the surface, and that is the effect that we have chosen to pursue in this work.

\section{DEFINING PRINCIPAL DIRECTIONS}

At any non-spherical point on a curved surface, there will be a single direction, orthogonal to the surface normal, in which the magnitude of the normal curvature is maximum and this is referred to as the first principal 
direction [10]. One can easily compute the principal directions and principal curvatures at any point on an isovalue surface in a three-dimensional volume from the first and second derivatives of the surrounding voxel values; details of such algorithms have been previously published [19][13] and will not be repeated here.

\section{RELATED WORK}

The idea that we might effectively communicate surface shape by explicitly representing the principal directions and principal curvatures on a surface has its precedents. Frobin and Hierholzer [7] computed the principal directions and principal curvatures on height surfaces defined in discretely sampled, acquired data of the human back, and displayed them as a pattern of cross-hairs on a two-dimensional grid, and Sander and Zucker [27] computed and displayed principal direction vectors on surface patches in three-dimensional data.

A number of different techniques have been recently proposed for "automatically" generating line-drawing surface representations in the style of pen-and-ink illustrators. Saito and Takahashi [25] defined a hachuring pattern based on the surface parameterization of a torus and applied it in proportion to the surface shading indicated by an illumination map. Although in this particular example the lines happen to follow the principal directions, this appears to occur as a fortuitous consequence of the symmetries of the form and may not be expected to occur in the general case. Winkenbach and Salesin [31] described methods for automatically generating pen-andink style renderings of architectural models in which resolution-independent stroke "textures" were applied to planar surfaces, and Salisbury et al. [26] created an interactive pen-and-ink style drawing program in which, among other things, the orientation of the individual elements of a higher-level stroke could be defined to follow the direction of the intensity gradient in a two-dimensional reference image.

\section{IMPLEMENTING A PRINCIPAL DIRECTION TEXTURE}

\subsection{Our Driving Application}

The driving application for our work with transparent surfaces has been radiation therapy treatment planning, in which physicians need to understand the three-dimensional distribution of a proposed radiation dose in the fullest possible context of the three-dimensional patient anatomy. The twin objectives in treatment plan design are to maximize the probability of tumor control and to minimize the probability of normal tissue complications. It is usually impossible to simultaneously optimize both of these functions within a single dose distribution, however, and determining the appropriateness of any particular plan requires the subjective weighing, by an experienced clinician, of complicated trade-offs between numerous criteria, both quantifiable and unquantifiable. Providing the physician with a clear display of the three-dimensional spatial relationship between the target volume and selected isointensity surfaces of radiation dose could help facilitate such judgements. Our goal is to improve the effectiveness with which the shape of an enclosing dose surface and its depth distance from an underlying target volume can be represented in both static and dynamic images and when viewed either monocularly or in stereo. We were primarily concerned in this work with designing an appropriate texture, and do not wish to suggest that we believe the particular implementation described below to be optimal. It is however, extremely straightforward and several wellknown methods can be employed to improve its computational efficiency.

\subsection{Defining the Individual Texture Elements}

Our method of applying a texture of oriented, opaque short strokes to a transparent isointensity surface is implemented within the framework of a raycasting volume renderer [16]. The locations, directions and lengths of the individual strokes are predefined in a sequence of steps that currently take several minutes (2-5) to execute, after the extraction of the isosurface geometry, on an IBM RS/6000.

The first step is to determine the points on the surface around which each texture element will be centered. (It should be noted that standard solid texturing algorithms do not require such a procedure; the textures in these cases are defined independently of the surfaces to which they are applied. Although surface-independent textures can be simpler to define, easier to render and more flexible in terms of being applicable to an arbitrary number of different level surfaces in a given volume, it is not possible to explicitly portray specific shape features of an individual surface using such an approach.) We iteratively select, from an unordered list of the centerpoints of each of the tiny subvoxel-sized triangles generated by a marchingcubes [18] isosurface extraction program, points that are no closer than a specified minimum Euclidean distance in object space from any other surface point previously chosen in order to distribute the strokes as evenly as possible over the surface.

Once the texture element centerpoints have been defined, principal directions and principal curvatures are computed at each of these points based on the local greylevel values in the volume data and used to determine the orientations and lengths of the strokes. Each stroke is modelled as a solid "slab" that modifies the opacity of the areas of the surface that pass through it. The corner vertices of a stroke slab are specified by the points $v_{x, y, z}=$ $\left(p_{x} \pm l \vec{e}_{1}, p_{y} \pm w e_{2}, p_{z} \pm h \vec{e}_{3}\right)$, where $p_{x}, p_{y}$ and $p_{z}$ are the scalar coordinates of the centerpoint, $l, w$ and $h$ specify the half-length, half-width and half-height of the slab and $\vec{e}_{1}, \vec{e}_{2}$ and $\vec{e}_{3}$ are the unit vectors that define the principal frame (with $\vec{e}_{1}$ lying in the first principal direction, $\vec{e}_{2}$ in the second principal direction, and $\vec{e}_{3}$ in the direction of the surface normal). We try to define the value of $h$ to be large enough to allow each slab to contain the surface across its fullest possible extent without being so large that it opacifies the surface in unintended areas. The value of $w$ can be arbitrarily small, but as it gets below 
about 0.35 voxel units we have found that a greater amount of super-sampling needs to be done to ensure that the strokes are adequately represented. The value of $l$ is defined as $l=l_{0}\left|k_{1} / k_{\max }\right|$, where $l_{0}$ is the maximum stroke length (specified by the user), $k_{1}$ is the magnitude of the principal curvature in the stroke direction, and $k_{\max }$ is a scaling factor that approximates the maximum of the magnitudes of the principal curvatures over all points on the isovalue surface.

The decision to vary the lengths of the strokes according to the magnitude of the surface curvature in the stroke direction was based on the premise that the boldness of the indication of a specific direction on a surface should be a reflection of the "importance" of that particular direction. Where a surface is relatively flat (or relatively spherical), any tiny perturbation could cause enormous changes in the orientations of the principal directions. Particularly in the case of flat surfaces, it makes little sense to strongly indicate one specific direction over any other. The use of longer strokes appears to be more warranted in the case of locally spherical regions, despite the instability of the orientations of the principal directions there, because the longer lines seem more capable of aptly conveying the rapidly changing nature of the surface orientation.

Although there is a fair amount of latitude possible in the specification of various parameters such as interelement spacing, element width, and maximum element length, we did not find it particularly profitable to vary the values of these parameters in search of an "optimal" combination. In general, we judged thinner strokes to be preferable to thicker strokes (as long as the strokes were not so thin that they occupied only a fraction of the width of a display pixel), and favored setting the minimum interelement distance to a value large enough to avoid as much as possible having multiple slabs overlap each other.

\subsection{Applying the Texture to the Surface}

Once their geometry is defined, the texture stokes still need to be applied to the transparent surface, and there are several different ways in which this can be done.

If the strokes are wide enough, they can be scanconverted into a volume opacity mask and during volume rendering the opacity of the isosurface at any point can be interpolated from the values in this volume. The primary advantage of such a technique would be that after the initial overhead of scan-conversion, multiple images of a textured surface can be rendered relatively quickly. The overriding disadvantage of this approach (and the reason that we do not use it) is that the crispness of the individual strokes can be severely compromised by the intermediate discretization step.

The approach that we prefer to use is to pass the geometrical definition of the individual strokes to the volume rendering program and, during raycasting, keep track of the intersections of each ray with the polygons bounding each slab. In this way we can simply and accurately determine whether or not any given ray/surface intersection point lies within an opacifying slab. By tagging each of the slab polygons with a label indicating the slab to which it belongs, we are able to gracefully handle the case of intersecting slabs and more robustly check for errors that can infrequently occur when a ray grazes the edge of a slab and an odd number of ray/slab intersections is registered. The primary disadvantage of this approach is the large amount of time that needs to be spent testing for possible intersections of a viewing ray with each of the slab-bounding polygons, but a number of different techniques [8] can be used to improve the efficiency of these computations.

\section{RESULTS, EMPIRICAL COMPARISONS WITH ALTERNATIVE APPROACHES}

Each of the images that we show in this section depicts a semi-transparent isointensity surface of radiation dose superimposed over an opaque treatment region. For clarity we have adopted a rendering style in which we show only the first occurrence of any particular transparent surface along each ray. Although this approach may be more appropriate in some situations than in others, for the purposes of consistency and to facilitate comparisons between the different texturing methods we have chosen to adopt it uniformly.

Figure 2 shows examples of the application of a principal direction texture to transparent isointensity surfaces in several different datasets, to give an impression of the kinds of results one may get when applying this method to surfaces of various different shapes.

An objective determination of the relative merits of adding a principal direction texture to a transparent surface, as opposed to adding no texture or using an alternative texture definition, needs to be based on controlled observer experiments that measure the ability of observers to make shape and depth judgements that depend on a simultaneous understanding of the layered surfaces, and we have been pursuing such work [12].

In this section, however, we intend only to provide, through empirical comparisons, some informal insights into the different natures of the various texturing techniques and do not mean to suggest that the utility of any of these approaches can be definitively inferred from an inspection of the images given here.

\subsection{Stroke Textures}

Figure 3-upper left shows a plain transparent dose surface enclosing an opaque tumor volume. Figure 3-upper right shows the same dataset, but with the external surface rendered opaquely. In figure 3-lower left, only the portions of the transparent surface covered by the principal direction texture are rendered with full opacity. To gain some insight into the relative merits of choosing the stroke directions and lengths in this particular manner, as opposed to some other, one may compare this image to the image in figure 3-lower right, in which the same layered surfaces are rendered with a stroke texture in which the element orientation is randomly defined and element length is constant. 

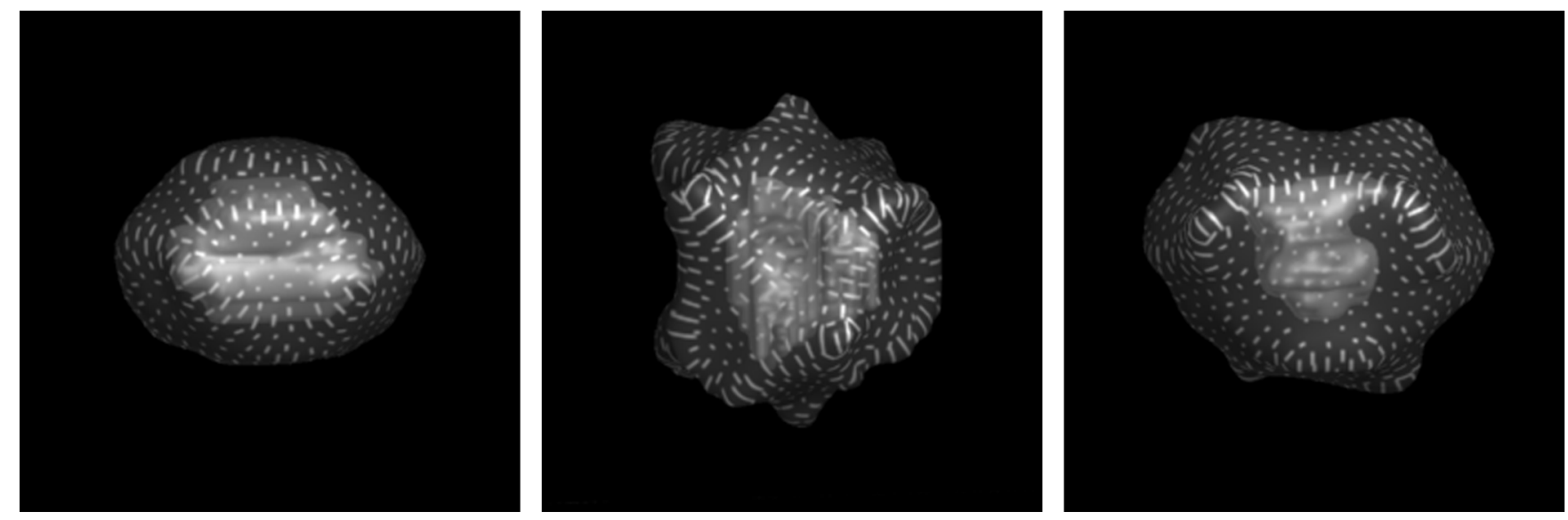

Figure 2: Principal direction texture applied to several different transparent isointensity surfaces of radiation dose.
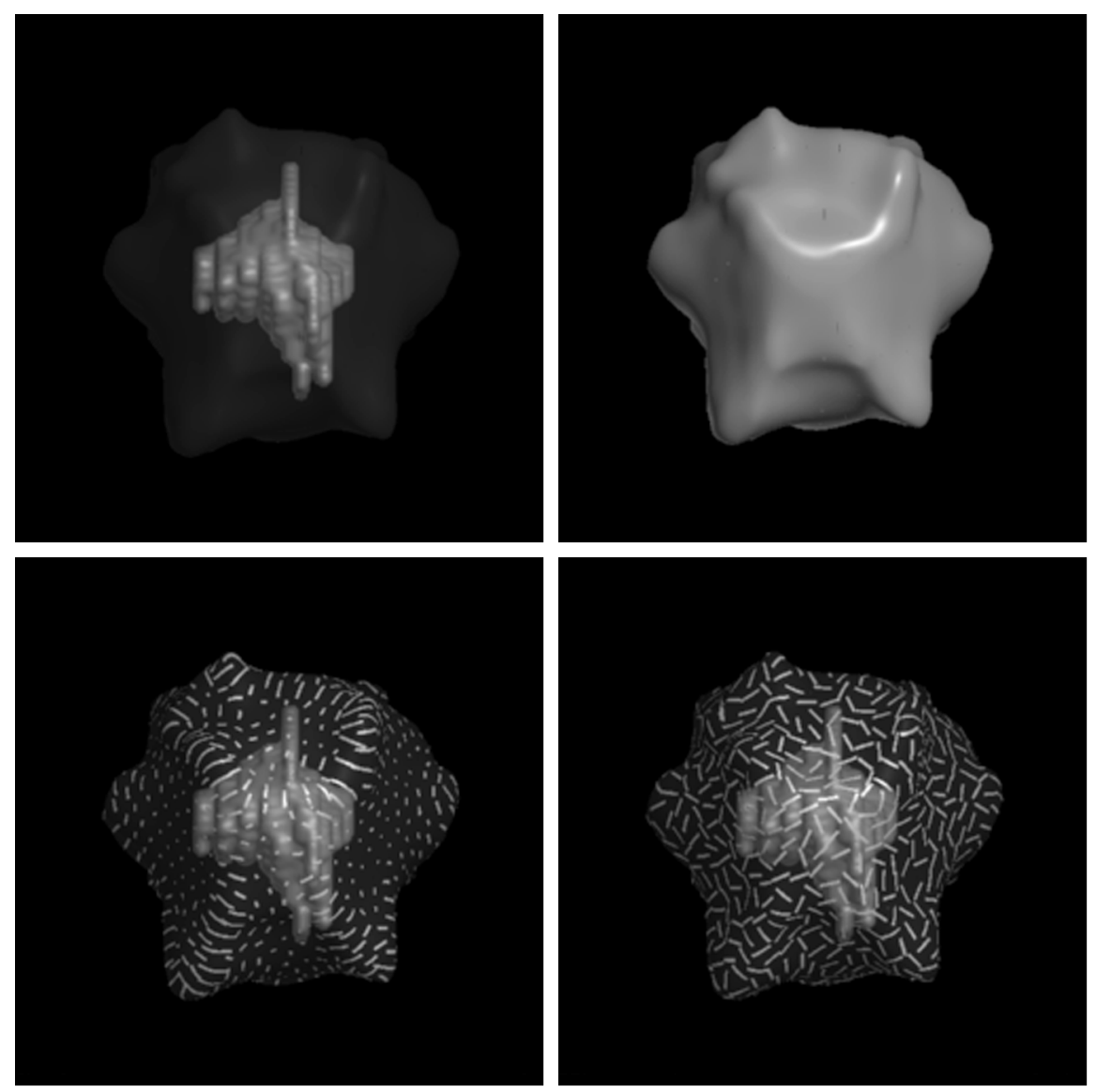

Figure 3: Each of these images depicts an isointensity surface of radiation dose surrounding an opaque treatment region. Upper left: transparent outer shell. Upper right: opaque outer shell. Lower left: transparent outer shell with principal direction texture. Lower right: transparent outer shell textured with randomly-oriented constant length strokes. 

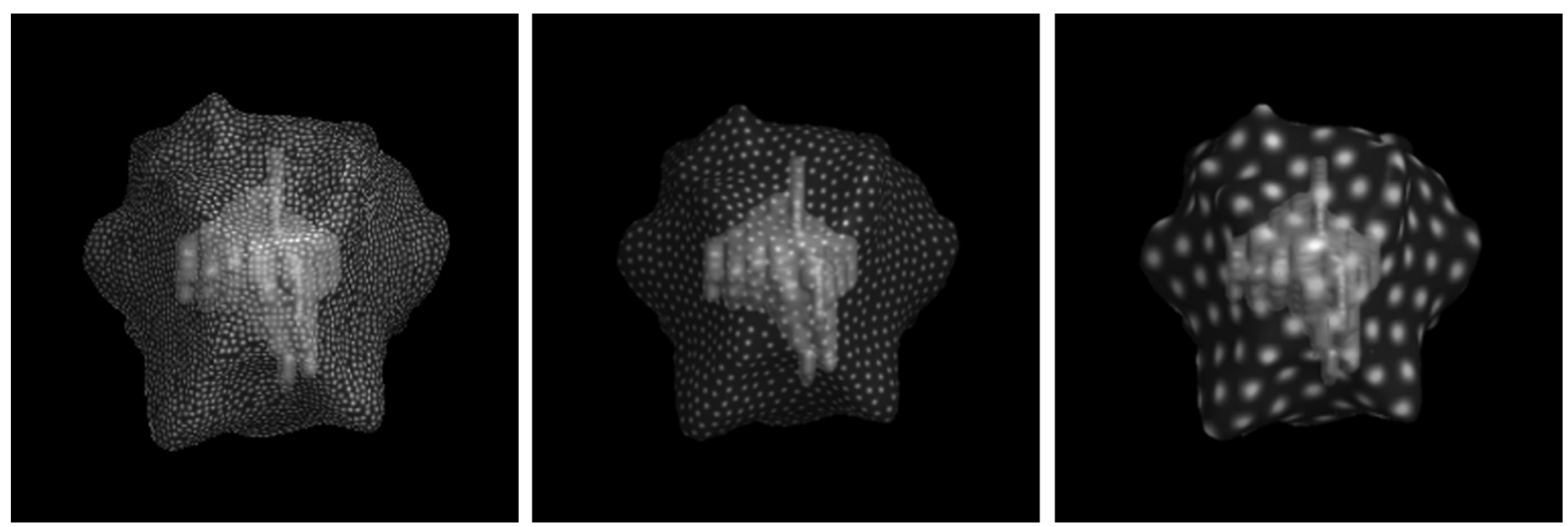

Figure 4: Spot textures of various sizes and spacings.
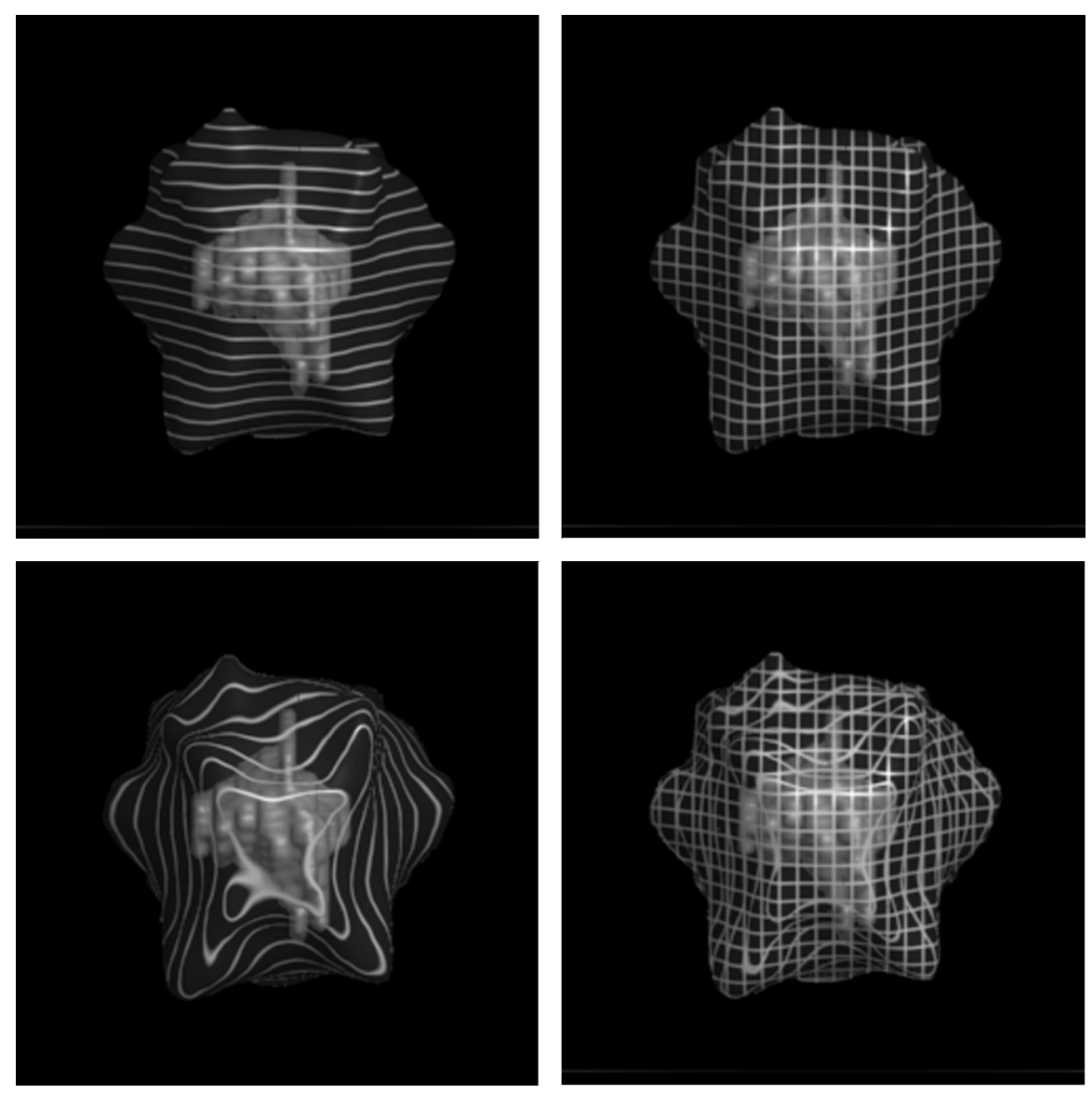

Figure 5: Contour line textures. 


\subsection{Spot Textures}

Figure 4 gives an example of the types of results that are achieved when circularly symmetric Gaussians rather than oriented slabs are used to modulate the opacity of the same transparent surfaces. The element centerpoints in each of these cases are defined using the same method described above for the stroke texture, so that they will be evenly spaced across the transparent surface; qualitatively different (and greatly inferior) results are achieved if the spherical texture elements are specified to lie at evenlyspaced points within the volume.

To appreciate the differences in the quality of the surface shape description provided by spot textures as opposed to principal direction strokes, it helps to imagine being asked, say, to reproduce the depicted surface in clay. While the spot texture gives good local information about the surface location, the strength of the principal direction texture lies in its ability to highlight the essential features of the structure of the surface shape - in this case the four ridges that meet at the central plateau.

\subsection{Contour Line Textures}

Figure 5 shows the results of texturing the same transparent surface with contour lines modeled by the intersection of the surface with solid planes evenly spaced along different combinations of object space axes. The particular appearance of the textures defined by any subset of grid lines, as in the upper left, upper right and lower left images, will obviously vary depending on the direction from which the surface is viewed; for example, the lines that appear horizontal in the upper left image would, if seen from above, resemble the concentric level curves of the image below it.

\section{CONCLUSIONS}

Transparency can be a useful device, in scientific visualization, for simultaneously depicting multiple layers of information. In computer-generated images, as in real life, however, transparent surfaces can be difficult to clearly see and also see through at the same time. By adding opaque texture elements to a layered transparent surface we may enable its three-dimensional shape and relative depth to be more easily and accurately perceived, and a texture pattern that explicitly conveys perceptually relevant characteristics of surface shape may be especially effective. Line orientation has long been regarded by artists and illustrators as being of significant importance in conveying the shape of objects in two-dimensional drawings, and lines that "follow the form" are believed to portray an object's shape particularly well. We have described a method for texturing transparent surfaces with uniformly distributed opaque short strokes, locally oriented in the direction of greatest normal curvature and having length proportional to the magnitude of the curvature in the stroke direction, and have provided an opportunity for empirical comparisons that may offer slight insight into the relative strengths and weaknesses of this surface texturing approach.

\section{ACKNOWLEDGMENTS}

This work was supported by NIH grant \# PO1 CA47982, and was performed as a part of the dissertation research of the first author, who received valuable advice in this effort from Frederick Brooks, Christina Burbeck, Julian Rosenman and Mary C. Whitton. Marc Levoy provided the volume rendering platform within which we implemented the isosurface extraction and surface texturing functions, and Jim Chung provided the implementation of the marching cubes algorithm that we use for the surface triangulation. The radiation therapy data is courtesy of Dr. Julian Rosenman, UNC Hospitals.

\section{REFERENCES}

[1] Blake, Andrew, Heinrich H. Bülthoff and David Sheinberg. "Shape from Texture: Ideal Observers and Human Psychophysics", Vision Research, vol. 33, no. 12, 1993, pp. 1723-1737.

[2] Blake, Andrew and Heinrich Bülthoff. "Shape from Specularities: computation and psychophysics", Philosophical Transactions of the Royal Society of London, B, vol. 331, 1991, pp. 237-252.

[3] Blinn, James F. and Martin E. Newell. "Texture and Reflection in Computer Generated Images", Communications of the ACM, vol. 19, no. 10, 1976, pp. 542-547.

[4] Bruno, Nicola and James E. Cutting. "Minimodularity and the Perception of Layout", Journal of Experimental Psychology: General, vol. 117, no. 2, 1988, pp. 161-170.

[5] Connolly, Michael L. "Solvent-Accessible Surfaces of Proteins and Nucleic Acids", Science, vol. 221, no. 4612,1983, pp. 709-713.

[6] Dooley, Debra and Michael F. Cohen. "Automatic Illustration of 3D Geometric Models: Surfaces", Visualization '90, pp. 307-313.

[7] Frobin, W. and E. Hierholzer. "Analysis of Human Back Shape Using Surface Curvatures", Journal of Biomechanics, vol. 15, no. 5, 1982, pp. 379-390.

[8] Glassner, Andrew. An Introduction to Ray Tracing, Academic Press, 1989.

[9] Guptill, Arthur. Rendering in Pen and Ink, WatsonGuptill Publications, 1976.

[10] Hilbert, David and S. Cohn-Vossen. Geometry and the Imagination, translated by P. Nemenyi, Chelsea Pub. Co., 1952.

[11]Hodges, Elaine R. S. The Guild Handbook of Scientific Illustration, Van Nostrand Reinhold, 1989.

[12] Interrante, Victoria. Illustrating Transparency: communicating the 3D shape of layered transparent surfaces via texture, Ph.D. thesis, University of North Carolina at Chapel Hill, 1996. 
[13] Interrante, Victoria, Henry Fuchs and Stephen Pizer. "Enhancing Transparent Skin Surfaces with Ridge and Valley Lines", Visualization '95, pp. 52-59.

[14] Koenderink, Jan J. and Andrea J. van Doorn. "Photometric Invariants Related to Solid Shape", Optica Acta, vol. 27, no. 7, 1980, pp. 981-996.

[15] Levoy, Marc, Henry Fuchs, Stephen Pizer, Julian Rosenman, Edward L. Chaney, George W. Sherouse, Victoria Interrante and Jeffrey Kiel. "Volume Rendering in Radiation Treatment Planning", First Conference on Visualization in Biomedical Computing, 1990, pp. 4-10.

[16]Levoy, Marc. "Display of Surfaces from Volume Data", IEEE Computer Graphics and Applications, vol. 8, no. 3, May 1988, pp. 29-37.

[17] Loechel, William E. Medical Illustration; a guide for the doctor-author and exhibitor, Charles C. Thomas, 1964.

[18]Lorensen, William and Harvey Cline. "Marching Cubes: A High Resolution 3D Surface Reconstruction Algorithm", Computer Graphics (proc. SIGGRAPH '87), vol. 21, no. 4, 1987, pp. 163-169.

[19] Monga, Olivier, Serge Benayoun and Olivier D. Faugeras. "From Partial Derivatives of 3D Density Images to Ridge Lines", Proceedings of the IEEE Computer Society Conference on Computer Vision and Pattern Recognition, 1992, pp. 354-359.

[20] Peachey, Darwyn R. "Solid Texturing of Complex Surfaces", Computer Graphics (proc. SIGGRAPH '85), vol. 19, no. 3, 1985, pp. 279-286.

[21]Photon Treatment Planning Collaborative Working Group. "Three-Dimensional Display in Planning Radiation Therapy: A Clinical Perspective", Int. J. Radiat. Oncology, Biol. Phys., vol. 21, 1991, pp. 7989.

[22] Pitz, Henry C. Ink Drawing Techniques, WatsonGuptill Publications, 1957.

[23]Rheingans, Penny. "Opacity-modulating Triangular Textures for Irregular Surfaces", proc. Visualization 96.

[24] Saito, Takafumi. "Real-time Previewing for Volume Visualization", 1994 Symposium on Volume Visualization, pp. 99-106.

[25]Saito, Takafumi and Tokiichiro Takahashi. "Comprehensible Rendering of 3-D Shapes", Computer Graphics (proc. SIGGRAPH '90), vol. 24, no. 4, pp. 197-206.

[26] Salisbury, Michael P., Sean E. Anderson, Ronen Barzel and David H. Salesin. "Interactive Pen-and-Ink Illustration", Computer Graphics (proc. SIGGRAPH ‘94), pp. 101-108.
[27] Sander, Peter T. and Steven W. Zucker. "Tracing Surfaces for Surfacing Traces", Proceedings of the First International Conference on Computer Vision, 1987, pp. 241-249.

[28] Schweitzer, Dino. "Artificial Texturing: An Aid to Surface Visualization”, Computer Graphics (proc. SIGGRAPH '83), vol. 17, no. 3, pp. 23-30.

[29] Stevens, Kent A. and Allen Brookes. "Probing Depth in Monocular Images", Biological Cybernetics, vol. 56, 1987, pp. 355-366.

[30] Sullivan, Edmund J. Line; an art study, Chapman \& Hall, 1922.

[31] Winkenbach, Georges and David H. Salesin. "Computer-Generated Pen-and-Ink Illustration", Computer Graphics (proc. SIGGRAPH '94), pp. 91100 .

[32] Young, Mark J., Michael S. Landy and Laurence T. Maloney. "A Perturbation Analysis of Depth Perception from Combinations of Texture and Motion Cues", Vision Research, vol. 33, no. 18, 1993, pp. 2685-2696.

[33]Zisserman, Andrew, Peter Giblin and Andrew Blake. "The Information Available to a Moving Observer from Specularities", Image and Vision Computing, vol. 7 , no. 1,1989 , pp. $38-42$. 